\title{
CONNECTING ORBITS FOR NONLINEAR PARABOLIC EQUATIONS*
}

\author{
NIKOLAI NADIRASHVILI ${ }^{\dagger}$
}

1. Introduction. We consider the scalar reaction diffusion equation

$$
u_{t}=f\left(x, u, u_{x}, u_{x x}\right)
$$

with $x \in S^{1}=\mathbb{R} / 2 \pi \mathbb{Z}, f \in C^{\infty}$ and

$$
C^{-1} \leq f_{u_{x x}} \leq C
$$

where $C$ is a positive constant. Let $u$ be a bounded solution of $(1)$ in $C^{1}\left(S^{1} \times(0, \infty)\right)$. Then as it follows from [1] and [2], the $\omega$-limit set $\omega(u)$ of the trajectory $u$ contains a solution of (1) which is periodic in $t$. If all periodic solutions of (1) are hyperbolic, then any bounded solution of equation (1) tends to a periodic solution (which can be in particular a steady state), as $t \rightarrow+\infty$. So it is interesting to study the connecting orbits between periodic solutions of (1). Let $L$ be a periodic hyperbolic orbit of (1). We denote by $W^{s}(L)$ and $W^{u}(L)$ the stable and unstable manifolds of $L$ respectively. For details of the definition and for general properties of stable and unstable manifolds of periodic solutions, refer to [3], [4], [5]. We denote by $M(L)=\operatorname{dim} W^{u}(L)$ the Morse index of $L$.

Let $L, L^{\prime}$ be periodic hyperbolic orbits of (1). If there exists a solution $u$ of (1) which is defined for all $t \in \mathbb{R}$ and such that $\alpha(u)=L$ and $\omega(u)=L^{\prime}$, then we say that $u$ connects $L$ and $L^{\prime}$, and we write in this case $L \rightarrow L^{\prime}$.

THEOREM 1. Let $L, L^{\prime}$ be periodic hyperbolic orbits of equation (1) and $L \rightarrow L^{\prime}$. Then $N(L) \geq M\left(L^{\prime}\right)$. If $L$ distinguishes from an equilibrium point then $M(L)>$ $M\left(L^{\prime}\right)$.

CoRollary. Let $L$ be a periodic hyperbolic orbit of (1) different from an equilibrium point. Then $L$ has no homoclinical trajectory.

REMARK. The detailed analysis of the connecting orbits for equation (1) with the Dirichlet boundary conditions was done in [6], [7], [8].

The main difference between the Dirichlet boundary condition and the periodic boundary condition for equations (1) and (2) is that in the case of the Dirichlet boundary condition equations (1) and (2) admit no limit cycles. result.

2. Some properties of linear parabolic equations and proof of the main

Let us consider a linear parabolic equation

$$
P u=\frac{\partial u}{\partial t}-a(t, x) \frac{\partial^{2} u}{\partial x^{2}}+b(t, x) \frac{\partial u}{\partial x}+c(t, x) u=0
$$

with $t \in \mathbb{R}, x \in S^{1}, a, b, c \in C^{\infty},\|a\|_{C^{k}},\|b\|_{C^{k}}:\|c\|_{C^{k}} \leq M(k), C^{-1} \leq a \leq C, C>0$. For $f \in C\left(S^{1}\right)$, we denote by $N f$ the number of changes in sign of the function $f$ on

\footnotetext{
*Received December 23, 1997, accepted for publication March 1, 1998.

†Institut for Problems of Information Transmission, Bolshoi Karetnyi 19, 101447 Moscow, Russia.
} 
$S^{1}$. So, if $f$ is a smooth function with simple zeros then $\# f^{(-1)}(0)=N f$, and $N f$ is an even number.

Let $v(t, x) \in C(\mathcal{D}), \mathcal{D}=\mathbb{R} \times S^{1}$, denote

$$
\begin{aligned}
N V\left(t_{0}\right) & =N V\left(t_{0}, x\right),\|V\|\left(t_{0}\right)=\left\|V\left(t_{0}, x\right)\right\|_{C\left(S^{1}\right)}, \\
U_{i} & =\left\{u \in C^{\infty}(\mathcal{D}), P u=0 \text { in } \mathcal{D}, N u(t) \equiv 2 i\right\} \cup\{0\}, \\
i & =0,1,2, \ldots .
\end{aligned}
$$

Theorem 2. For any $i$ the set $U_{i}$ is a linear space, $\operatorname{dim} U_{0}=1, \operatorname{dim} U_{i}=2$, $i=1,2, \ldots$. If $i<j, u_{i} \in U_{i}, u_{j} \in U_{j},\left\|u_{i}\right\|(0)=\left\|u_{j}\right\|(0)$ then for $t>0$

$$
\left\|u_{j}\right\|(t) \leq c_{j}^{\prime} e^{-c_{j} t}\left\|u_{i}\right\|(t)
$$

for $t<0$,

$$
\left\|u_{i}\right\|(t) \leq c_{j}^{\prime} e^{-c_{j} t}\left\|u_{j}\right\|(t)
$$

Here $c_{j}, c_{j}^{\prime}$ are positive constants. If $u, v$ is a basis in $U_{i}, i=1,2, \ldots$, then the level curves $u=0$ separates the level curves $v=0$. Let $u$ be a solution of the problem (3), then $N u(t) \leq i$ for all $t \in \mathbb{R}$ if and only if $u \in U_{0} \oplus \cdots \oplus U_{i}$.

The proof of Theorem 2 is based on the following lemmas.

LEMMA 1. ([9]). Let $f_{n}$ be a sequence of bounded functions on [0,1), continuous from the right, $f_{n} \not \equiv 0$, and $N f_{n} \leq k$. Then exists a real valued sequence $\alpha_{m}$ and subsequence $f_{n_{m}}$ such that, the sequence

$$
\alpha_{m} f_{n_{m}}
$$

is convergent, in the sense of distributions, to distribution $\bar{f} \not \equiv 0$ of order $\leq k$.

As a consequence of Lemma 1 we have

LemMA 2. Let $f_{n} \in C\left(S^{1}\right), N f_{n} \leq k$ for all $n=1,2, \ldots$. Then there exists a subsequence $n_{i}$ and real numbers $\alpha_{i}$ such that as $i \rightarrow \infty, \alpha_{i} f_{n_{i}}$ converges in the topology of the space of distributions $\mathcal{D}^{\prime}$ to a non-zero distribution of order less than $k$.

Lemma 3. ([10]). Let $u$ be a solution of (3), $t \in[0,1]$. Then $N u(t)$ is a nonincreasing function on $[0,1]$.

Lemma 4. ([11]). Let $u$ be a solution of (3), $t \in(0,1)$. Then for each $t \in(0,1)$, $N u(t)<\infty$.

Lemma 5. ([11]). Let $u$ be a solution of (3), $t \in(0,1), N u(t) \equiv i$. Then for each $t_{0} \in(0,1)$ the function $u\left(t_{0}, x\right)$ has exactly $i$ zeros on $S^{1}$, and each zero of $u\left(t_{0}, x\right)$ on $S^{1}$ is simple.

Lemma 6. Let $u$ be a solution of (3), $t>0, N u(t) \equiv k, 0<\tau<T$. Then

$$
\|u\|(\tau) /\|u\|(T)<C
$$

where $C=C(k, \tau, T, M(k))>0$.

Proof. We assume the contrary, namely, that there exists a sequence of parabolic equations of the type (3)

$$
P_{i} u_{i}=0 \text { in } S^{1} \times(0, \infty),
$$

$N u_{i}(t) \leq k$, and

$$
\left\|u_{i}\right\|(\tau) /\left\|u_{i}\right\|(T) \rightarrow \infty
$$


as $i \rightarrow \infty$. From Lemma 2 it follows that choosing convergent subsequences $P_{i_{m}}, u_{i_{m}}$ we obtain an equation

$$
P u=0 \text { in } S^{1} \times(0, \infty)
$$

such that $u \not \equiv 0$ on $S^{1} \times(0, T), u(T, \cdot) \equiv 0$ on $S^{1}$. This contradicts the theorem on the uniqueness of the solution of the inverse problem for parabolic equations [12].

LEMma 7. Let $u$ be a solution of (3), $t>0, N u(t) \equiv k$. Denote by $\alpha_{1}(t), \ldots$, $\alpha_{k}(t)$ the zeros of the function $u(t, \cdot)$ on $S^{1}$. Then there exists a constant $\beta=$ $\beta(k, M(k))>0$ such that

$$
\inf _{\substack{t>1 \\ 1 \leq i \leq k-1}}\left(\alpha_{i+1}(t)-\alpha_{i}(t)\right)>\beta
$$

Proof. We assume the contrary, namely, that there exists a sequence of parabolic equations of the type (3),

$$
P_{j} u_{j}=0 \text { in } S^{1} \times(0, \infty),
$$

$N u_{j}(t) \equiv k, j=1,2, \ldots$, and real numbers $t_{j}>1$ such that for some $i$

$$
\alpha_{i+1}^{j}\left(t_{j}\right)-\alpha_{i}^{j}\left(t_{j}\right) \rightarrow 0
$$

as $j \rightarrow \infty$, where $\alpha_{1}^{j}, \ldots, \alpha_{k}^{j}$ are zeros of the function $u_{j}$. Without loss of generality, we may assume that all $t_{j}=1$, and the coefficients of $P_{j}$ converge in $C^{k}$ to the coefficients of $P$, as $j \rightarrow \infty ;\left\|u_{j}\right\|(1)=1, j=1,2, \ldots$.

From Lemma 5 it follows that there is a subsequence $u_{j_{m}}$ which is convergent to the solution of the equation

$$
P u=0 \text { in } S^{1} \times(0,1) .
$$

From Lemmas 3 and 5 , it follows that $N u(t) \leq k-1$ for $t>1$, and there is a $t_{0}>1$ such that all zeros of the function $u\left(t_{0}, \cdot\right)$ are simple. As $u_{j_{m}}\left(t_{0}, \cdot\right) \rightarrow u\left(t_{0}, \cdot\right)$ in $C^{1}$ then there is such $u_{n}$ that $N u_{n}(t) \leq k-1$.

LEMMA 8. Let $u, v$ be solutions of (3), $t>0, N u(t) \equiv i, N u(t) \equiv j, i<j$, $\|u\|(1)=\|v\|(1)$.

Proof. Let us assume that for some $T>1\|v\|(T) /\|u\|(T)$ is sufficiently large. Then by Lemmas 5, 6, 7 for the sufficiently small $\varepsilon>0, N(u+\varepsilon v)(1)=i, N(u+$ $\varepsilon v)(T)=j$. As $i<j$ then our assumption contradicts Lemma 2 .

Lemma 9. Let $u, v$ be solutions of (3), $t>0, N u(t) \equiv i, N v(t) \equiv j, i<j$, $\|u\|(1)=\|v\|(1)$. Then there exists $T>1, T=T(j, M(j))$, such that

$$
\|u\|(T) /\|v\|(T)>2 \text {. }
$$

Proof. Let us assume the opposite. Then from Lemma 8 it follows that there is a sequence $t_{k} \rightarrow \infty,\|u\|\left(t_{k}\right) /\|v\|\left(t_{k}\right) \rightarrow s>0$ as $k \rightarrow \infty$. Further, we may assume that the following sequences are convergent in $C^{1}\left(S^{1}\right)$ :

$$
\begin{aligned}
& \frac{u\left(t_{k}, x\right)}{\|u\|\left(t_{k}\right)} \rightarrow \varphi, \\
& \frac{v\left(t_{k}, x\right)}{\|v\|\left(t_{k}\right)} \rightarrow \psi,
\end{aligned}
$$


as $k \rightarrow \infty$. Since $N \varphi=i, N \psi=j$, then for any $\varepsilon>0$ there is an $\alpha \in \mathbb{R}$ such that the distance between a pair of zeros of the function $\varphi+\alpha \psi$ is less than $\varepsilon$. So the same is true for the function $u\left(t_{k}, x\right)+\alpha s v\left(t_{k}, x\right)$ for sufficiently large $k$. The last statement contradicts Lemma 7.

LEMmA 10. For all $T>0, i=1,2, \ldots$ there exists a two-dimensional space $U(T, i)$ of solutions of the problem (2) in $S^{1} \times(-T, T)$ such that if $u \in U(T, i), u \not \equiv 0$, then $N u(t)=2 i, t \in(0, T)$. If $u, v$ is a basis in $U(T, i)$, then the level curves $u=0$ separates the level curves $v=0$.

Proof. 1. We define parabolic operators $P_{\theta}, \theta \in[0,1]$ on $S^{1} \times(-T, 3 T)$ by

$$
\begin{gathered}
P_{1}=\frac{\partial}{\partial t}-a_{1}(t, x) \frac{\partial^{2}}{\partial x^{2}}+b_{1}(t, x) \frac{\partial}{\partial x}+c_{1}(t, x), \\
a_{1}(t, x)= \begin{cases}a(t, x) & \text { for } x \in(-T, T], \\
-a(3 T-t, x) & \text { for } x \in(T, 3 T),\end{cases} \\
b_{1}(t, x)= \begin{cases}b(t, x) & \text { for } x \in(-T, T], \\
-b(3 T-t, x)-\frac{\partial}{\partial x} a(3 T-t x) & \text { for } x \in(T, 3 T),\end{cases} \\
c_{1}(t, x)= \begin{cases}c(t, x) & \text { for } x \in(-T, T], \\
c(3 T-t, x)-\frac{\partial}{\partial x} b(3 T-t, x)-\frac{\partial^{2}}{\partial x^{2}} a(3 T-t, x) & \text { for } x \in(T, 3 T),\end{cases} \\
P_{0}=\frac{\partial}{\partial t}-\frac{\partial^{2}}{\partial x^{2}},
\end{gathered}
$$

Let us consider the problem

$$
\left\{\begin{array}{l}
P_{\theta} u=0 \quad \text { in } S^{1} \times(-T, 3 T) \\
u(0, x)=g(x) .
\end{array}\right.
$$

Denote by $L^{\theta}$ the linear operator $L^{\theta}: g(x) \rightarrow u(3 T, x)$. For any $\theta \in[0,1], L^{\theta}$ is a selfadjoint operator in $L_{2}\left(S^{1}\right)$.

2. Let $g$ be an eigenfunction of $L^{\theta}, u$ be a solution of (6). Then $N u(-T)=$ $N u(3 T)$, and hence by Lemma 5 the zeros of the function $u\left(t_{0}, x\right), t_{0} \in[-T, 3 T]$ are simple.

3. Denote by $\lambda_{1}^{\theta}>\lambda_{2}^{\theta} \geq \lambda_{3}^{\theta} \geq \cdots$ the eigenvalues of $L^{\theta}, \theta \in[0,1]$. Then $\lambda_{1}^{0}=1$, $\lambda_{2}^{0}=\lambda_{3}^{0}=e^{-4 T}, \lambda_{4}^{0}=\lambda_{5}^{0}=e^{-16 T}, \ldots$ The eigenfunctions of $L^{0}$ are: $1, \sin n x, \cos n x$, $n=1,2, \ldots$. Since the eigenvalues $\lambda_{n}^{\theta}$ and their eigenvector subspaces are continuously dependent on the parameter $\theta$ then from 2 it follows that for any $i=1,2, \ldots$ and $\theta \in[0,1]$ the operator $L^{\theta}$ has exactly two linear independent eigenfunctions $\varphi^{\theta}, \psi^{\theta}$ (or two-dimensional subspace if $\lambda_{2 i}^{\theta}=\lambda_{2 i+1}^{\theta}$ ) such that $N \varphi^{\theta}=N \psi^{\theta}=i$.

4. Let $u, v, w$ be solutions of (6) with $g=\varphi^{\theta}, \psi^{\theta}, h=a \varphi^{\theta}+b \psi^{\theta} \not \equiv 0, a, b \in \mathbb{R}$. We prove that the function $w$ has simple zeros. Denote $L \varphi^{\theta}=\alpha \varphi^{\theta}, L \psi^{\theta}=\beta \psi^{\theta}$. If $\alpha=\beta$, the statement follows from Assertion 2 .

If $\alpha \neq \beta$, we assume the contrary. Then by Lemma $5 N \omega(-T)>N \omega(3 T)$. Since

$$
\left(L^{\theta}\right)^{k}(h)=\alpha^{k} \alpha \varphi^{\theta}+\beta^{k} b \psi^{\theta},
$$

$k \in \mathbb{Z}$, then for sufficiently large $k$

$$
N\left[\left(L^{\theta}\right)^{k}(h)\right]=N\left[\left(L^{\theta}\right)^{-k}(h)\right]=i .
$$


This contradicts the inequality $N w(-T)>N w(3 T)$.

5. From 4 it follows that $N w(t) \equiv i, t \in[-T, 3 T]$ and that $\left(u^{\theta}\right)^{2}+\left(v^{\theta}\right)^{2}>0$ on $S^{1} \times[-T, 3 T]$. Hence we may consider $u^{1}, v^{1}$ as a basis in $U(T, i)$.

Proof of Theorem 2. Let $U(T, i)$ be defined as in Lemma 10. By Lemma 6 we can choose sequences $\alpha_{k}, T_{k} \in \mathbb{R}, T_{k} \rightarrow+\infty$ as $k \rightarrow \infty$ such that $U\left(T_{k}, i\right) \rightarrow U_{i} \not \equiv 0$ in $\mathcal{D}$ as $k \rightarrow \infty$. If $u \in U_{i}$ then by Lemmas 5 and $6, N u(t) \equiv 2 i, t \in \mathbb{R}, i=1,2, \ldots$. The inequalities (4) and (5) follow from Lemma 9.

Let $u$ be a solution of $(2), t \in \mathbb{R}$, and $N u(t) \equiv i$. We prove that $u \in U_{i}$. Assume the contrary, namely, that $u, u_{i}, v_{i}$ are linear independent, where $u_{i}, v_{i} \in U_{i}$. Then there is a linear combination $w=a u+b u_{i}+c v_{i}$ such that $w(0,0)=\frac{\partial}{\partial x} w(0,0)=0$. By Lemma $5 N w(-1)>N w(1)$. If $N w(1)<i$ then by Lemma $9,\|u\|(t),\left\|u_{i}\right\|(t)$, $\left\|v_{i}\right\|(t)=o(\|w\|(t))$ as $t \rightarrow+\infty$, which is impossible. If $N w(1) \geq i$ then $N w(-1)>i$ and $\|u\|(t),\left\|u_{i}\right\|(t),\left\|v_{i}\right\|(t)=o(w(t))$ as $t \rightarrow-\infty$, which is also impossible. So, $u \in U_{i}$. Equality $\operatorname{dim} U_{0}=1$ is evident. If $u \in U_{0} \oplus \cdots \oplus U_{i}$, the inequality $N u(t) \leq i$ follows from (5).

Let $u$ be a solution of $(3), t \in \mathbb{R}, N u(t) \leq i$. First we prove that $u \in U_{0} \oplus \cdots \oplus U_{i+1}$. Let us assume the contrary. Then there is $v \in U_{0} \oplus \cdots \oplus U_{i+1}$ such that $N(u+v)(0)>$ $i+1$. By Lemma $9\|u\|(t),\|v\|(t)=o(\|u+v\|(t))$ as $t \rightarrow-\infty$ which is impossible. So, $u=\alpha v+\beta w$, with $v \in U_{0} \oplus \cdots \oplus U_{i}, w \in U_{i+1}$. If $\beta \neq 0$, then from inequality (5) it follows that for sufficiently large $T>0, N u(-T)=i+1$. Hence $u \in U_{0} \oplus \cdots \oplus U_{i}$, and so Theorem 2 is proved.

If in Theorem 2 we consider Dirichlet or Neumann boundary conditions the situation becomes more simple. Let us consider the problem

$$
\begin{cases}P u=0 & \text { in } \mathbb{R} \times[0,1], \\ u(t, 0)=u(t, 1)=0 . & \end{cases}
$$

Let us denote by $V_{i}$ the set of solutions of (7) such that for $v \in V_{i}, N v(t) \equiv i$.

Theorem 3. For any $i$ the set $V_{i}$ is a one-dimensional linear space. If $i<j$, $v_{i} \in V_{i}, v_{j} \in V_{j},\left\|v_{i}\right\|(0)=\left\|v_{j}\right\|(0)$, then for $t>0$

$$
\left\|v_{j}\right\|(t) \leq c_{j}^{\prime} e^{-c_{j} t}\left\|v_{i}\right\|(t)
$$

and for $t<0$

$$
\left\|v_{i}\right\|(t) \leq c_{j}^{\prime} e^{-c_{j} t}\left\|v_{j}\right\|(t)
$$

where $c_{j}, c_{j}^{\prime}>0$.

Evidently it is possible to prove Theorem 3 by the same reasoning as in the proof of Theorem 2. We don't need Theorem 3 for the following, but it is interesting to compare both cases.

Proof of Theorem 1. Let $u$ be a connecting orbit between $L$ and $L^{\prime}$. Let us consider the function

$$
v_{T}(t, x)=\frac{\partial u / \partial t}{\|\partial u / \partial t\|(T)}(t+T, x) .
$$

Then $v_{T}(t, x)$ converges to the exponential growing solution of the variational equation on $L$ as $T \rightarrow-\infty$ and $v_{T}(t, x)$ converges to the exponentially decreasing solution of the variational equation on $L^{\prime}$ as $T \rightarrow+\infty$. By Lemma $3, k=N \frac{\partial u}{\partial t}(-\infty) \geq N \frac{\partial u}{\partial t}(+\infty)=$ $m$. So by Theorem 2

$$
\operatorname{dim} W^{n}(L) \geq 2 k, \operatorname{dim} W^{u}\left(L^{\prime}\right) \leq 2 m .
$$


Let us assume now that $L$ is the orbit of the periodic solution $w$ of (1) and $\partial w / \partial t \not \equiv 0$. Then by Theorem $2, N \frac{\partial w}{\partial t} \geq k$. Thus $\operatorname{dim} W^{u}(L) \geq 2 k+1$ and hence $M(L)>M\left(L^{\prime}\right)$. Theorem 1 is proved.

\section{REFERENCES}

[1] B. Fiedler, J. Mallet-Paret, A Poincaré-Bendixson theorem for scalar reaction diffusion equations, Arch. Rat. Mech. Anal. 107 (1989), 325-345.

[2] N.S. NAdiRAshVili, On the dynamics of nonlinear parabolic equations, Dokl. Acad. Nauk USSR 309 N6 (1989); English transl. in Soviet Math. Dokl. 40 (1990), N3, 636-639.

[3] M. Hirsh, C. Pugh, M. Shub, Invariant manifolds, Lecture Notes in Math., Springer-Verlag 583 (1977).

[4] D. HenRy, Geometric theory of semilinear parabolic equations, Lecture Notes in Mathematics, Springer-Verlag 840 (1981).

[5] A.V. BABIN, M.I. VISHIK, Attractors of partial differential evolution equations and estimates of their dimension, Uspekhi Mat. Nauk 38 N4 (1983), 133-187; English transl. in Russian Math. Surveys 38:4 (1983).

[6] P. BRunovský, B. FiedleR, Number of zeros on invariant manifolds in reaction-diffusion equations, Nonlinear Analysis 10 (1986), 179-193.

[7] P. BRUnovskÝ, B. FIEdLER, Heteroclinic connections of stationary solutions of scalar reactiondiffusion equations, Banach Center Publications, Warsaw 19 (1987).

[8] P. BRunovskÝ, B. FIEDLER, Connecting orbits in scalar reaction diffusion equations II. The complete solution, J. Diff. Eq. 81 (1989), 106-135.

[9] N.S. NADIRASHVILI, Metric properties of eigenfunctions of the Laplace operator on mani-folds, Ann. Inst. Fourier (Grenoble) 41 (1991), 259-265.

[10] K. NiCKEL, Gestaltaussagen über Lösungen parabolischer Differentialgleichungen, J. reine angew. Math. 211 (1962), 78-94.

[11] S. Angenent, The zero set of a solution of a parabolic equation, J. reine angew. Math. 390 (1988), 79-96.

[12] J.L. Lions, B. Malgrange, Sur l'unicité rétrograde dans les problèmes mixes parabolique, Math. Scand. 8 (1960), 277-286. 